\title{
Novel injectable and self-setting composite materials for bone defect repair
}

\author{
Qian $\mathrm{Xu}^{1,2 \dagger}$, Jiulong Liang ${ }^{3 \dagger}$, Haipeng Xue ${ }^{3}$, Yun'en $\mathrm{Liu}^{3}$, Lei $\mathrm{Cao}^{2,4}, \mathrm{Xiaowu} \mathrm{Li}^{1^{*}}$, Jing $\operatorname{Tian}^{3^{*}}$ and \\ Xing Zhang ${ }^{2,4^{*}}$
}

\begin{abstract}
Although calcium sulfate bone cements possess favorable characteristics of excellent clinical handling and osteoconductivity, the rapid degradation and poor bioactivity limit their wide applications. This study reports a type of novel injectable and self-setting composite materials for bone defect repair. Magnesium substituted tricalcium phosphate nanoparticles $(43.8 \pm 9.0 \mathrm{~nm})$ and calcium sulfate hemihydrate micro-size particles $(5-21 \mu \mathrm{m})$ were mixed with a polyvinyl alcohol solution with optimal compositions to obtain the composite materials, which showed reasonable initial setting time (11.7-19.2 min) and suitable compressed strength (2.28-6.33 MPa). By utilizing magnesium powder as a porogen, macro-pores $(>100 \mu \mathrm{m})$ together with micro-pores were created in the final product after setting. In addition, MC3T3E1 cells extended well and exhibited numerous lamellipodia and long filopodia when cultured with the composite materials, indicating that they had no cytotoxicity. The in vivo results indicated that the injectable composite materials could achieve bone defect repair, when implanted in beagle femoral condyle defects for 10 months. Our results show that the new injectable composite materials are biocompatible and biodegradable, which possess great potential for bone defect repair.
\end{abstract}

Keywords: nanoparticles, magnesium substitution, injectable materials, self-setting, bone defect repair

\section{INTRODUCTION}

A variety of injectable bone cements have been extensively utilized for bone defect repair and regeneration [1-4]. Among them, calcium phosphate cements (CPCs) and calcium sulfate cements (CSCs) exhibit advantageous functions, such as appropriate self-setting time, good biocompatibility, and osteoconductivity $[5,6]$. However, the degradation rate of CPCs with a final composition of hydroxyapatite (HA) is relatively slow, at approximately 1-2 wt.\% per year, due to the low solubility of $\left(\mathrm{Ca}_{5^{-}}\right.$ $\left(\mathrm{PO}_{4}\right)_{3} \mathrm{OH}:-\log \left(K_{\mathrm{sp}}\right)=58.4$, at $\left.25^{\circ} \mathrm{C}\right) \quad[7,8]$. Porous HA blocks (Bonfil ${ }^{\mathbb{R}}$, Mitsubishi Materials, Chichibu, Japan) with a porosity of $70 \%$ and a mean pore diameter of $280 \mu \mathrm{m}$ (sintered at $900^{\circ} \mathrm{C}$ ) were replaced by newly formed bone after implantation into a bone defect $(8 \mathrm{~cm} \times$ $3 \mathrm{~cm}$ ) of the patient's ilium for 79 months [9]. There is a risk of late deformity due to the inadequate degradation of the bone substitute material [10]. On the other hand, CSCs show rapid degradation, with a higher solubility $\left(\mathrm{CaSO}_{4}:-\log \left(K_{\mathrm{sp}}\right)=3.5\right.$, at $\left.25^{\circ} \mathrm{C}\right)$ approximately $1-2$ months in vivo, which is much faster compared with bone regeneration [11-13]. In some cases, the bone defects remained unfilled while the CSCs implants were completely degraded, which led to the fracture of bone tissue $[1,14]$. Thus, an appropriate degradation rate should be achieved for injectable bone materials that can ideally match with new bone regeneration.

Relatively poor osteoinductivity or osteogenic capacity constitutes another major limitation for clinical applications of current ceramic bone cements [15]. Previous studies reveal that the presence of $\mathrm{Mg}^{2+}$ is likely beneficial to the new bone growth, and promotes the proliferation and differentiation of osteoblastic progenitor cells $[16,17]$. $\mathrm{Mg}^{2+}$ is able to enter cells through the transient receptor

\footnotetext{
${ }^{1}$ Department of Materials Physics and Chemistry, School of Materials Science and Engineering, Key Laboratory for Anisotropy and Texture of Materials, Ministry of Education, Northeastern University, Shenyang 110819, China

${ }^{2}$ Institute of Metal Research, Chinese Academy of Sciences, Shenyang 110016, China

${ }^{3}$ General Hospital of Northern Theater Command, Shenyang 110016, China

${ }^{4}$ School of Materials Science and Engineering, University of Science and Technology of China, Hefei 230026, China

† These authors contributed equally to this work.

* Corresponding authors (emails: xwli@mail.neu.edu.cn (Li X); soldier_chn@163.com (Tian J); xingzhang@imr.ac.cn (Zhang X))
} 
potential melastatin (TRPM) family TRPM7. $\mathrm{Mg}^{2+}$ $\left(10 \mathrm{mmol} \mathrm{L}^{-1}\right)$ can promote osteogenesis in human bone marrow stromal cells (hBMSCs) through the activation of canonical Wnt signaling pathway, in which the downstream protein $\beta$-catenin transfers into the nucleus and up-regulates the direct target genes, LEF1 and Dkk1 [18]. $\mathrm{Mg}^{2+}$ (5-10 $\mathrm{mmol} \mathrm{L}^{-1}$ ) can also improve the osteogenesis in hFOB1.19 human osteoblast cells by up-regulating the expression of Runx2 and ALP via the TRPM7/PI3K signaling pathway [19]. Díaz-Tocados et al. [20] demonstrated that $\mathrm{Mg}^{2+}\left(1.2\right.$ or $\left.1.8 \mathrm{mmol} \mathrm{L}{ }^{-1}\right)$ in pro-osteogenic medium enhanced the osteogenesis, mineralization and differentiation of bone marrow mesenchymal stem cells (MSCs) by activating the Notch1 signaling pathway.

$\mathrm{Mg}^{2+}$ can be supplied by using $\mathrm{Mg}$-substituted calcium phosphate $(\mathrm{CaP})$ materials, in which nanoparticles are more favorable for cellular uptake and easier to be degraded compared with micro-size particles [21-23]. Meanwhile, $\mathrm{CaP}$ nanoparticles possess unique properties because of their chemical and crystallographic similarity to the inorganic components of bone [22]. A previous study reported that $\mathrm{CaP}$ nanoparticles of approximately 50-100 nm promoted the proliferation of MSCs compared with micro-size particles of approximately $1-2 \mu \mathrm{m}$, due to their nanoscale topography [23]. Herein, $\mathrm{Mg}^{2+}$ was doped into the tricalcium phosphate nanoparticles as bioactive ion sources.

In this study, Mg-substituted tricalcium phosphate (hereafter referred to as TCMP) nanoparticles were synthesized, and further mixed with calcium sulfate hemihydrate $(\mathrm{CSH})$ micro-size particles to obtain solid phases, in order to adjust the degradation time and improve the bioactivity in vivo. Injectable and self-setting composites were developed by combining the solid phase with a liquid phase of polyvinyl alcohol (PVA) solution. The setting time of these composite materials and compressive strength of the resultant products were measured. In vitro cytotoxicity was evaluated by co-culture of the resultant products with MC3T3-E1 cells. Moreover, bone defect repair with the injectable composite materials was performed by using a beagle femoral defect model.

\section{EXPERIMENTAL SECTION}

\section{Preparation of TCMP nanoparticles}

$\mathrm{CSH}$ of analytical grade was purchased from ACROS Organics (Acros, Fukuoka, Spain). The particle size was in the range of $5-21 \mu \mathrm{m}(n=30$, three photographs were selected and 10 particles were evaluated in each photograph). Other chemicals and reagents were obtained from
Sinopharm Chemical Reagent (Shanghai, China), unless otherwise noted.

A mixed solution of $\mathrm{Ca}\left(\mathrm{NO}_{3}\right)_{2} \cdot 4 \mathrm{H}_{2} \mathrm{O}\left(0.9 \mathrm{~mol} \mathrm{~L}^{-1}\right)$ and $\mathrm{Mg}\left(\mathrm{NO}_{3}\right)_{2} \cdot 6 \mathrm{H}_{2} \mathrm{O}\left(0.1 \mathrm{~mol} \mathrm{~L}^{-1}\right)$ was prepared by dissolving the chemicals in deionized water. The above solution was then added into a $\left(\mathrm{NH}_{4}\right)_{2} \mathrm{HPO}_{4}$ solution $\left(0.6 \mathrm{~mol} \mathrm{~L}^{-1}\right)$, and the $\mathrm{pH}$ was adjusted to 9.5 with ammonium hydroxide and stirred at $500 \mathrm{rmin}^{-1}$, which was further aged at $80^{\circ} \mathrm{C}$ for $4 \mathrm{~h}$. The precipitate product was thoroughly rinsed with deionized water thrice, and further dried at $120^{\circ} \mathrm{C}$ for $12 \mathrm{~h}$. The dried TCMP powder was screened with a 200 -mesh sieve.

\section{Fabrication of injectable and self-setting composite materials}

The solid phase of injectable composite materials was obtained by mixing TCMP and $\mathrm{CSH}$ powders. $\mathrm{Mg}$ granules screened below 200 mesh were used as the porogen. Different amounts of $\mathrm{Mg}$ granules (0, $1 \mathrm{wt} . \%$, 5 wt.\%) were added to the solid phases, hereafter referred to as TCMP-CSH, TCMP-CSH-1\%Mg, and TCMP-CSH$5 \% \mathrm{Mg}$ samples. The compositions of the solid phases for the composite materials are listed in Table 1. A 5 wt.\% polyvinyl alcohol (PVA) solution as the liquid phase was mixed with the solid phase at the weight ratio of the liquid phase/solid phase of 0.5 , which was placed in a mildew incubator (MJ-II, Shanghai Drawell Scientific Instrument Co., Ltd., Shanghai, China) at $37^{\circ} \mathrm{C}$ with a relative humidity of approximately $95 \%$ for further setting.

\section{Characterization of chemical composition and sample morphology}

The crystal phase of samples was analyzed by X-ray diffraction (Rigaku, Tokyo, Japan) with a $2 \theta$ range from $20^{\circ}$ to $70^{\circ}$, at a voltage of $40 \mathrm{kV}$, and a current of $100 \mathrm{~mA}$ with monochromated $\mathrm{CuKa}$ radiation of $1.5418 \AA$. Fourier transform infrared spectrometry (FTIR, Cary 630, Agilent Technologies, USA) was conducted in the range of $400-4000 \mathrm{~cm}^{-1}$ by using the attenuated total reflection (ATR) mode.

Sample morphology was observed by an LEO Supra 35

Table 1 Chemical compositions of the solid phases for different composite materials

\begin{tabular}{cccc}
\hline Samples & CSH (wt.\%) & TCMP (wt.\%) & Mg (wt.\%) \\
\hline TCMP-CSH & 50 & 50 & 0 \\
TCMP-CSH-1\%Mg & 50 & 49 & 1 \\
TCMP-CSH-5\%Mg & 50 & 45 & 5 \\
\hline
\end{tabular}


field emission scanning electron microscope (FESEM, Carl Zeiss AG, Jena, Germany) operated at an accelerating voltage of $20 \mathrm{kV}$. Prior to observation, samples were sputter-coated with gold for $60 \mathrm{~s}$ by using a sputter coating instrument (Cressington, Watford, England). Chemical analysis of the TCMP powder was performed by inductively coupled plasma-optical emission spectrometry (ICP-MS, Prodigy, Leeman Laboratories, Hudson, USA). For the ICP-MS test, $10 \mathrm{mg}$ of powder was dissolved in $10 \mathrm{~mL}$ of $2 \mathrm{wt} . \%$ nitric acid solution, and $1 \mathrm{~mL}$ of the above solution was diluted with $50 \mathrm{~mL}$ of deionized water. The elemental compositions were analyzed by energy dispersive X-ray spectroscopy (EDX, INCAX 7582, Oxford Instruments, Abingdon, UK). The bright field images of TCMP powder were observed by a Tecnai G2 transmission electron microscope (TEM, FEI, Hillsboro, USA) at $200 \mathrm{kV}$ with a double-tilt holder (Gatan, Pleasanton, USA). The electron diffraction patterns of the particles were recorded by using a selected-area aperture (10 $\mu \mathrm{m}$ diameter).

\section{Setting time and mechanical property}

The setting time was recorded according to the ASTM C191-18a standard by using a Vicat apparatus (Shanghai Shenrui Test Equipment Manufacturing Co., Ltd., Shanghai, China). The solid and liquid phases were mixed for $30 \mathrm{~s}$ to form a homogenous paste. The paste was filled into a stainless-steel mold $(6 \mathrm{~mm}$ diameter, $12 \mathrm{~mm}$ height), which was then placed in a mildew incubator at $37^{\circ} \mathrm{C}$ with approximately $95 \%$ relative humidity. A needle of $1 \mathrm{~mm}$ diameter under a load of 300-g force was used to determine the setting time. The initial setting time was recorded when an indentation of approximately $1 \mathrm{~mm}$ depth was obtained. The final setting time was achieved when there was no prominent indentation on the surface. Five replicates were tested for each sample.

For mechanical tests, the pastes in cylindrical molds ( $6 \mathrm{~mm}$ diameter, $12 \mathrm{~mm}$ height) were placed in a humidified mildew incubator at $37^{\circ} \mathrm{C}$ with a relative humidity of approximately $95 \%$ for $48 \mathrm{~h}$ to achieve complete setting. The resultant hardened samples were polished with sandpaper (2000 grit) to obtain a height of $10 \mathrm{~mm}$ and a diameter of $6 \mathrm{~mm}$. The mechanical strength of these samples was measured by quasi-static compression tests by using a Bose ELF3230 machine (TA Instruments, Eden Prairie, USA) at a crosshead speed of $0.02 \mathrm{~mm} \mathrm{~s}^{-1}$ until fracture occurred. The maximum value of compressive force was recorded based on the curve of load and displacement. The compressive strength was calculated as Force $_{\max } /\left(\pi d^{2} / 4\right)$, where $d$ is the diameter of the sample.
Five replicates were tested for each sample.

\section{Porosity measurement}

The cylindrical samples (6 mm diameter, $10 \mathrm{~mm}$ height) of the composite materials (TCMP-CSH, TCMP-CSH-1\% $\mathrm{Mg}$, and TCMP-CSH-5\%Mg) were used for the porosity measurement (three replicates each). The porosity was estimated by the Archimedes drainage method. The original weight $\left(M_{1}\right)$ of each sample in air was measured. Samples were then immersed in absolute ethanol for $24 \mathrm{~h}$. The surface liquid was dried by a filter paper and the final weight $\left(M_{2}\right)$ was recorded. The porosity $(P)$ of each sample was determined by the following:

$P=\frac{M_{2}-M_{1}}{\rho V}$,

where $M_{1}$ is the original weight, $M_{2}$ is the final weight after immersion, $\rho$ is the density of absolute ethanol and $V$ is an apparent volume that can be calculated from the geometry of cylindrical sample.

\section{In vitro cell culture}

MC3T3-E1 cells were purchased from the Cell Bank of the Chinese Academy of Sciences (Shanghai, China). MC3T3-E1 cells were cultured in regular culture medium containing serum-free a-MEM (Hyclone, GE Healthcare, Chicago, USA) supplemented with $10 \% v / v$ fetal bovine serum (FBS, Gibco, Fisher Scientific, Pittsburgh, USA), and $1 \%$ antibiotic/antimycotic (Gen-View Scientific Inc., Calimesa, USA) in a humidified $\mathrm{CO}_{2}$ incubator (Thermo Fisher Scientific, Waltham, USA) containing $5 \% \mathrm{CO}_{2}$ at $37^{\circ} \mathrm{C}$. The culture medium was refreshed every three days.

The cell biocompatibility was assessed according to the ISO 10993-5 standard with a minor adjustment. The hardened samples were immersed in the serum-free $\alpha$ MEM medium $\left(0.1 \mathrm{~g} \mathrm{~mL}^{-1}\right)$ with rotation at $80 \mathrm{r} \mathrm{min}^{-1}$ in an orbital shaker (Jing Hong Laboratory Instrument Co., Ltd., Shanghai, China) at $37^{\circ} \mathrm{C}$ for $24 \mathrm{~h}$. The extracts were then filtered through $0.22 \mu \mathrm{m}$ filter membranes, and supplemented with $10 \% v / v$ FBS and $1 \% v / v$ antibiotic/ antimycotic to obtain the extraction medium. The concentrations of $\mathrm{Mg}^{2+}$ and $\mathrm{Ca}^{2+}$ in the extracts were evaluated by using the ICP-MS. The TCMP-CSH, TCMP$\mathrm{CSH}-1 \% \mathrm{Mg}$, and TCMP-CSH-5\%Mg samples were immersed in a phosphate buffer saline (PBS) solution at $37^{\circ} \mathrm{C}$ for $24 \mathrm{~h}\left(0.01 \mathrm{~g} \mathrm{~mL}^{-1}\right)$, and the $\mathrm{Mg}^{2+}$ and $\mathrm{Ca}^{2+}$ concentrations in the extracts were measured by ICP-MS.

MC3T3-E1 cells were seeded at a density of $2.5 \times 10^{3}$ cells/well in 96-well plates, and cultured with $100 \mu \mathrm{L}$ regular culture medium per well. After incubation at $37^{\circ} \mathrm{C}$ 
for $12 \mathrm{~h}$ in a $\mathrm{CO}_{2}$ incubator, the culture medium was replaced with the extraction medium for further culture of 1,3 , and 5 days. The cells were rinsed with $a$-MEM medium and replaced by $100 \mu \mathrm{L}$ regular culture medium, to eliminate the influence of $\mathrm{Mg}^{2+}$ in the extracts on the assay of Cell Counting Kit-8 (CCK-8) [24]. A $10 \mu \mathrm{L}$ CCK8 (Gen-View Scientific Inc., Calimesa, USA) solution was subsequently added to each well, which was further incubated for $2.5 \mathrm{~h}$. The absorbance was measured at a wavelength of $450 \mathrm{~nm}$ by using a microplate reader (Multiskan Go, Thermo Fisher Scientific, Waltham, USA). The cell viability was analyzed by the mean of optical density (OD) values and standard deviation (SD).

MC3T3-E1 cells were seeded on the TCMP-CSH, TCMP-CSH- $1 \% \mathrm{Mg}$, and TCMP-CSH- $5 \% \mathrm{Mg}$ samples at a density of $2 \times 10^{4}$ cells/well ( $1 \mathrm{~mL}$ per well). The samples were gently rinsed by PBS after cell seeding for $24 \mathrm{~h}$, and immobilized in $4 \%$ paraformaldehyde for $2 \mathrm{~h}$, which were further dehydrated in a graded series of ethanol solutions $(50 \%, 70 \%, 90 \%, 95 \%$ and $100 \%)$ for $10 \mathrm{~min}$ each and dried with hexamethyldisilazane (HMDS) for $20 \mathrm{~min}$. Sample morphology was observed by an LEO Supra 35 FESEM (Carl Zeiss AG, Jena, Germany) operated at an accelerating voltage of $20 \mathrm{kV}$.

\section{Fluorescent imaging}

MC3T3-E1 cells were first inoculated into six-well plates at the initial density of $4 \times 10^{4}$ cells/well ( $4 \mathrm{~mL}$ per well) for $24 \mathrm{~h}$. The cell medium was replaced by $4 \mathrm{~mL}$ of the extraction medium when the cell confluence reached approximately $80 \%$. Cells were gently washed by PBS and fixed with $4 \%$ polyformaldehyde for $20 \mathrm{~min}$, followed by PBS washing for three times. A fluorescein phalloidin (FITC-phalloidin) solution $\left(10 \mu \mathrm{g} \mathrm{mL}^{-1}\right.$ ) (Mao Kang Biotechnology Co., Ltd., Shanghai, China) was added to the wells and stained for $50 \mathrm{~min}$. The samples were subsequently washed with PBS three times and stained with a 4',6-diamidino-2-phenylindole (DAPI) solution $\left(100 \mathrm{ng} \mathrm{mL}^{-1}\right.$ ) to counterstain the cell nuclei for $15 \mathrm{~min}$. These samples were finally washed with PBS before observation under an Eclipse Ti inverted fluorescence microscope (Nikon Instruments Inc., Tokyo, Japan).

\section{In vivo bone defect repair}

Four healthy beagles $(\sim 12-15 \mathrm{~kg})$ were anesthetized with propofol $\left(1 \mathrm{~mL} \mathrm{~kg}^{-1}\right.$ of animal weight) via intravenous injection. After routine disinfection, hind legs were shaved and an incision was procured to expose the femur condyle. A cylindrical bone defect $(8 \mathrm{~mm}$ diameter, $10 \mathrm{~mm}$ depth) was created. The defect sites were ran- domly filled with TCMP-CSH or TCMP-CSH-5\%Mg samples, or unfilled as the control group. The skin wound was sutured, and all animals were injected with penicillin $\left(25,000 \mathrm{U} \mathrm{kg}^{-1}\right)$ post-operation for the first three days.

Digital radiographs for the implantation sites of the hind legs with the beagles under anesthesia were taken post-operation for 10 months by using an X-ray medical machine (UD150B-30, Shimadzu Production Institute, Tokyo, Japan). Bone repair ability was detected by X-ray computed tomography (Discovery CT750 HD, GE Healthcare, Chicago, USA). The femur specimens were cut-off around the implanted sample, and were further decalcified by using $10 \%$ ethylenediaminetetraacetic acid (EDTA) decalcification (Lianmai Biotechnology, Shanghai, China). The decalcified bone sections were stained with hematoxylin and eosin (H\&E) for histopathological analysis. All procedures for animal experiments were approved by the ethics committee of the General Hospital of Northern Theater Command.

\section{RESULTS AND DISCUSSION}

\section{Characterization of TCMP nanoparticles}

The bioactivity of scaffolds was critically important for bone defect repair. CPCs with micro-size HA particles showed relatively low bioactivity. On the other hand, CaP nanoparticles were found to promote the proliferation of MSCs compared with micro-size particles [23]. Therefore, TCMP nanoparticles were synthesized in this study in order to develop bioactive injectable composite materials.

The morphology of a TCMP nanoparticle under TEM is shown in Fig. 1a. The corresponding electron diffraction pattern of the particle (Fig. 1b) indicated the crystalline phase of TCMP corresponding well with $\beta$-TCP crystals, including ( $\left.\begin{array}{lll}1 & 1 & 0\end{array}\right),\left(\begin{array}{lll}2 & \overline{1} & 0\end{array}\right)$, and ( $\left.\begin{array}{lll}1 & \overline{2} & 0\end{array}\right)$ crystalline planes with $\left[\begin{array}{lll}0 & 0 & 1\end{array}\right]$ zone axis. There was obvious aggregation of TCMP nanocrystals (Fig. 1c). The particle size was found to be approximately $43.8 \pm 9.0 \mathrm{~nm}$ in diameter $(n=45$, three photographs were selected and 15 nanoparticles were evaluated in each photograph). The concentric speckle diffraction rings in the selected area electron diffraction (SAED) pattern diagram (Fig. 1d) corresponded well with $\beta$-TCP crystals, including lattice planes of (lllll $110 .\left(\begin{array}{lll}0 & 2 & 4\end{array}\right)$, and ( $\left.\begin{array}{lll}3 & 0 & 0\end{array}\right)$. The XRD pattern of the as-synthesized TCMP powder (Fig. 1e) included main peaks of (2 14 ), ( $\left.\begin{array}{lll}0 & 2 & 10\end{array}\right)$ and (2 20 ), corresponding well with $\beta$-TCP (JCPDF card \#09-0169); whereas the diffraction peaks for TCMP shifted to larger angles due to the substitution of $\mathrm{Ca}^{2+}$ (radius $=1.0 \AA$ ) by $\mathrm{Mg}^{2+}$ (radius $=0.72 \AA$ ) [25]. An obvious contraction of crystal 


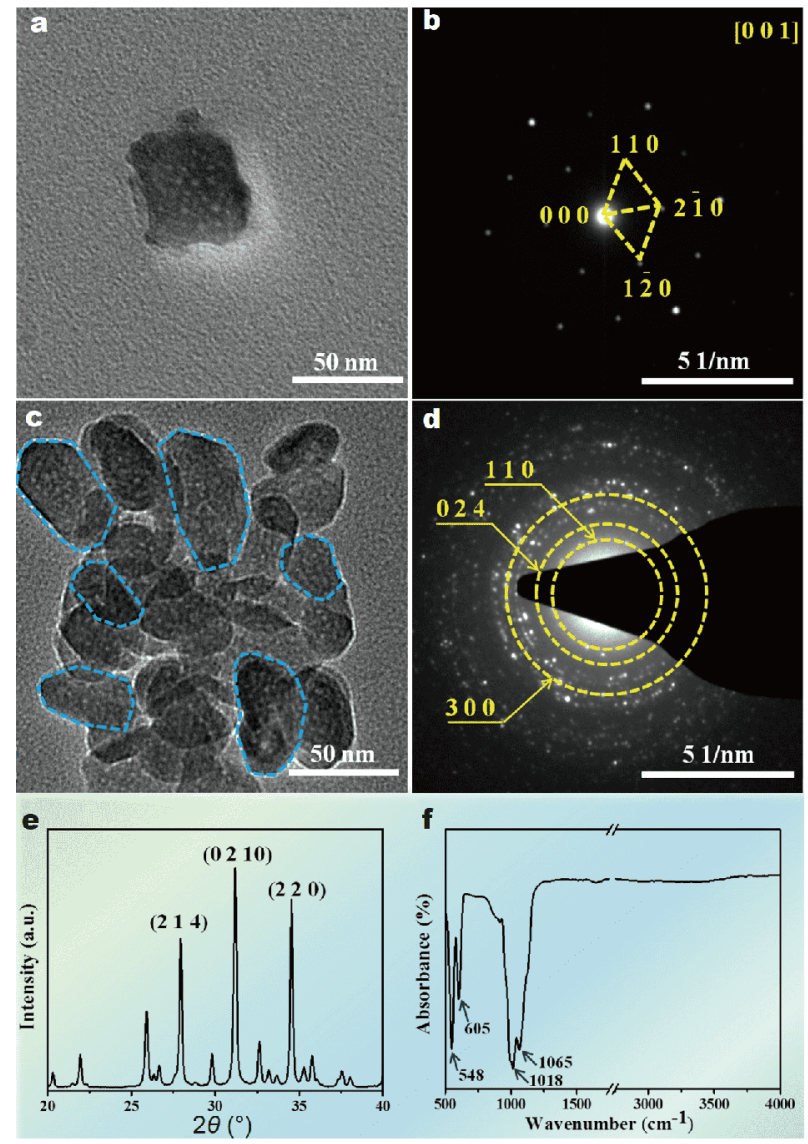

Figure 1 Characterization of the synthetic TCMP nanoparticles. (a, c) Bright field images of the synthetic TCMP nanoparticles; (b) corresponding electron diffraction pattern of TCMP with [ $\left[\begin{array}{lll}0 & 0 & 1\end{array}\right]$ zone axis; (d) corresponding SAED pattern of TCMP crystals; (e) XRD pattern of the TCMP sample; (f) FTIR spectrum of the TCMP sample.

lattices was observed from $a=10.492 \AA, c=37.380 \AA$ to $a=10.373 \AA$ and $c=37.203 \AA$, respectively, based on the fitting analysis by using the software Jade 6.5. The peaks at 548 and $605 \mathrm{~cm}^{-1}$ in the FTIR spectrum of TCMP (Fig. 1f) were assigned to the bending mode of $\mathrm{O}-\mathrm{P}-\mathrm{O}$ linkage in the phosphate group, while the bands at 1018 and $1065 \mathrm{~cm}^{-1}$ were ascribed to the asymmetric stretching of the $\mathrm{P}-\mathrm{O}$ bond of the phosphate group. The ICP result showed that the final $\mathrm{Ca} / \mathrm{Mg}$ molar ratio was $9.42 \pm 0.15$.

\section{Development of injectable and self-setting composite materials}

The solid and liquid phases were thoroughly mixed to develop injectable composite materials. The solid phase consisted of CSH and TCMP powder, with or without $\mathrm{Mg}$ granules as the porogen. The diffraction pattern of $\mathrm{CSH}$ included main peaks from $\left(\begin{array}{lll}0 & 2 & 0\end{array}\right),\left(\begin{array}{lll}4 & 0 & 0\end{array}\right)$ and $\left(\begin{array}{lll}2 & 0 & 4\end{array}\right)$
(Fig. 2a), corresponding well with JCPDF card \#41-0224 (peak positions indicated by the green vertical lines). The FTIR spectrum of the CSH sample is shown in Fig. $2 \mathrm{~b}$. The bands at 590 and $662 \mathrm{~cm}^{-1}$ were assigned to the bending vibration of the $\mathrm{SO}_{4}{ }^{2-}$ group, while the bands at $1081 \mathrm{~cm}^{-1}$ were attributed to the stretching mode of the $\mathrm{S}-\mathrm{O}$ group. The absorption peaks at 3548 and $3605 \mathrm{~cm}^{-1}$ were ascribed to the stretching vibration of the $\mathrm{O}-\mathrm{H}$ group. The peak at $1618 \mathrm{~cm}^{-1}$ was assigned to the bending vibration of the $\mathrm{H}-\mathrm{O}-\mathrm{H}$ group. The XRD pattern and FITR result of the TCMP-CSH-5\%Mg sample after final setting are shown in Fig. $2 \mathrm{c}$ and d. TCMP and a partial amount of $\mathrm{Mg}$ were retained in the sample. Calcium sulphate dihydrate (CSD) (Fig. 2c, red lines, JCPDF card \#33-0311) was present in the final product, which was produced by the reaction between $\mathrm{CSH}$ and water. The FTIR spectrum of the final product (Fig. 2d) further confirmed the reaction from CSH to CSD. The bands at 550 and $598 \mathrm{~cm}^{-1}$ were assigned to the bending vibration of the $\mathrm{SO}_{4}{ }^{2-}$ group. The absorption peaks at 3393 and $3520 \mathrm{~cm}^{-1}$ were attributed to the stretching vibration of the $\mathrm{O}-\mathrm{H}$ group. The peak at $1618 \mathrm{~cm}^{-1}$ from the bending vibration of the $\mathrm{H}-\mathrm{O}-\mathrm{H}$ group proved the presence of $\mathrm{H}_{2} \mathrm{O}$ molecules in CSD [26]. The initial and final setting times of the injectable composite materials are shown in Fig. 2e. The initial and final setting times of the TCMP$\mathrm{CSH}$ sample were $11.7 \pm 0.5$ and $14.5 \pm 0.5 \mathrm{~min}$, respectively. The addition of $\mathrm{Mg}$ powder into TCMP-CSH-1\% $\mathrm{Mg}$ and TCMP-CSH-5\%Mg samples, however, resulted in a substantial increase of the initial and final setting times, $12.7 \pm 0.8$ and $16.8 \pm 0.8 \mathrm{~min}$ for TCMP-CSH- $1 \% \mathrm{Mg}$ samples, and $19.2 \pm 0.8$ and $27.5 \pm 1.0 \mathrm{~min}$ for TCMP-CSH$5 \% \mathrm{Mg}$ samples, respectively. The average compressive strength for the composite materials of TCMP-CSH, TCMP-CSH-1\%Mg, and TCMP-CSH-5\%Mg were $6.33 \pm$ $1.21,2.71 \pm 0.39$, and $2.28 \pm 0.20 \mathrm{MPa}$, respectively (Fig. $2 \mathrm{f}$ ). The morphologies of TCMP-CSH, TCMP-CSH-1\%Mg, and TCMP-CSH-5\%Mg samples are shown in Fig. S1. The samples expanded with the addition of $\mathrm{Mg}$ powder as the porogen after the final setting, and the volume increased with the increase of $\mathrm{Mg}$ content.

Suitable setting time is a requisite criterion for injectable bone grafts. Injectable composite materials must be extruded and injected into defects prior to the initial setting time [27]. CSH can solidify by mixing with water for $5 \mathrm{~min}$ through a slight exothermic reaction [28]. The injectable calcium sulphate $\left(\mathrm{MIIG}^{\circledR} \mathrm{X}_{3}\right.$, Wright Medical Technology, Inc., Massachusetts, USA) must be extruded within 3 min due to the fast setting, resulting in a short time window for surgery $[29,30]$. Other commercial bone 

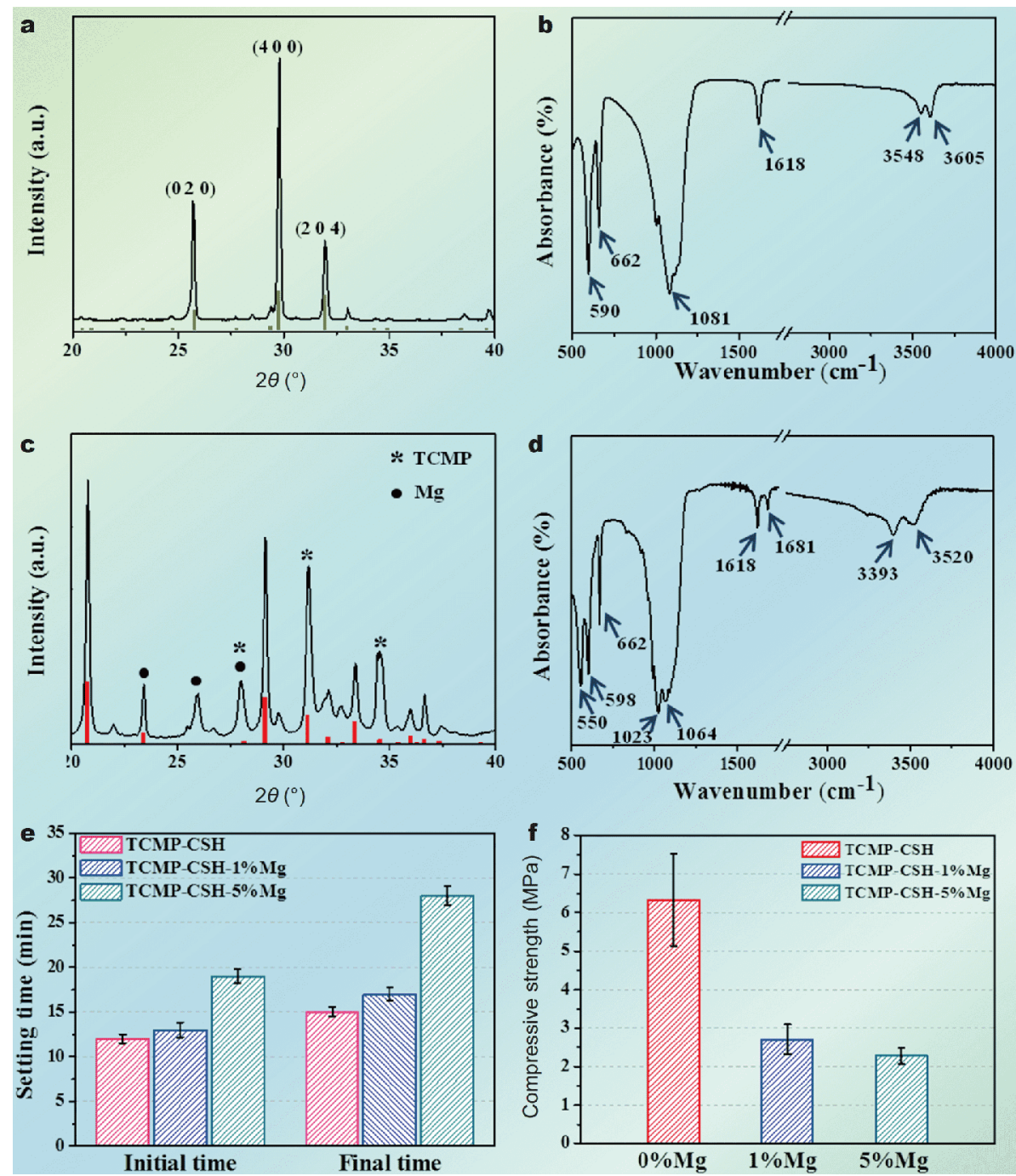

Figure 2 Characterization of the injectable composite materials. (a) XRD pattern and (b) FTIR spectrum of a CSH sample; (c) XRD pattern of the TCMP-CSH-5\%Mg sample after final setting; (d) FTIR spectrum of the TCMP-CSH sample after final setting; (e) initial and final setting times for the TCMP-CSH, TCMP-CSH-1\%Mg and TCMP-CSH-5\%Mg samples; (f) compressive strength of the TCMP-CSH, TCMP-CSH- $1 \% \mathrm{Mg}$ and TCMP$\mathrm{CSH}-5 \% \mathrm{Mg}$ samples after final setting.

cements, such as Norian ${ }^{\circledR}$ SRS $^{\circledR}$ (Norian Corporation, California, USA), Biobone ${ }^{\circledR}$ (ETEX Corporation, Massachusetts, UK) and Bone Source ${ }^{\circledR}$ (Stryker Howmedica osteonics, Mississippi, USA), showed the initial setting time in the range of 10-20 min [5,31]. Our injectable composite materials exhibit initial setting times in the range of 12-19 min, which are suitable for surgery operation.

The compressive strength of the TCMP-CSH, TCMPCSH-1\%Mg and TCMP-CSH-5\%Mg samples after setting were $6.33 \pm 1.21,2.71 \pm 0.39$ and $2.28 \pm 0.20 \mathrm{MPa}$, close to that from cancellous bone (2-12 MPa) [32]. The average porosity of the three samples was $34.4 \% \pm 3.8 \%, 43.7 \%$ $\pm 2.0 \%$ and $52.3 \% \pm 2.3 \%$, respectively (Fig. S2). Therefore, the porosity increased and the compressive strength decreased with the increase of $\mathrm{Mg}$ content in the composite materials. Previous research revealed that CPC cements with different compositions and porosities (50\%-84\%) showed the compressive strength ranging from 2 to $20 \mathrm{MPa}$ [33-35]. Therefore, the compressive strength of our products is close to those from porous CPC cements, which can be utilized for small bone defect-filling in nonload bearing cases.

\section{Morphology and microstructure of composite materials}

Microstructures of the TCMP-CSH, TCMP-CSH-1\%Mg, 
and TCMP-CSH-5\%Mg samples after complete setting are shown in Fig. 3. The TCMP-CSH sample exhibited relatively dense morphology (Fig. 3a), with a large number of micro-pores $(<10 \mu \mathrm{m})$. With the increase of $\mathrm{Mg}$ content, there was an obvious increase in the total number of pores and pore size. Besides micro-pores, a certain amount of meso-pores approximately $10-100 \mu \mathrm{m}$ were found in the TCMP-CSH-1\%Mg sample (Fig. 3b). Remarkably, macro-pores $(>100 \mu \mathrm{m})$ together with mesopores and micro-pores (Fig. 3c) were found in the TCMP-CSH-5\%Mg group, likely due to the reaction of $\mathrm{Mg}$ (porogen) with $\mathrm{H}_{2} \mathrm{O}$. Rod-like CSD particles uniformly distributed in the composite materials (Fig. 3d-f), consisting of the major matrix of the final products. The EDX results further demonstrated the existence of CSD and TCMP particles (Fig. 3g, h).

The porous structures of scaffolds are beneficial to bone reconstruction, which facilitate the cell adhesion, nutrient transportation, blood vessel growth, and bone regeneration [36-38]. When the pore size is less than $10 \mu \mathrm{m}$, this allows circulation of body fluid. When the pore size is between $10-75 \mu \mathrm{m}$, penetration of fibrous tissue is allowed [39]. When the pore size is between 75 and $100 \mu \mathrm{m}$, the growth of unmineralized osteoid tissue is facilitated. When the pore size is larger than $100 \mu \mathrm{m}$, the growth of mineralized bone is promoted [39]. In this study, $\mathrm{Mg}$ granules were used as a porogen for the injectable composite materials, which produced both micro- and macro-pores due to formation of $\mathrm{H}_{2}$ bubbles by the reaction of $\mathrm{Mg}$ and water during the paste preparation process [40]. The number and size of the pores can be modulated by the Mg content in the composite material. The chemical reaction of $\mathrm{Mg}$ particles and water is depicted as

$\mathrm{Mg}+2 \mathrm{H}_{2} \mathrm{O} \rightarrow \mathrm{Mg}(\mathrm{OH})_{2}+\mathrm{H}_{2}$.

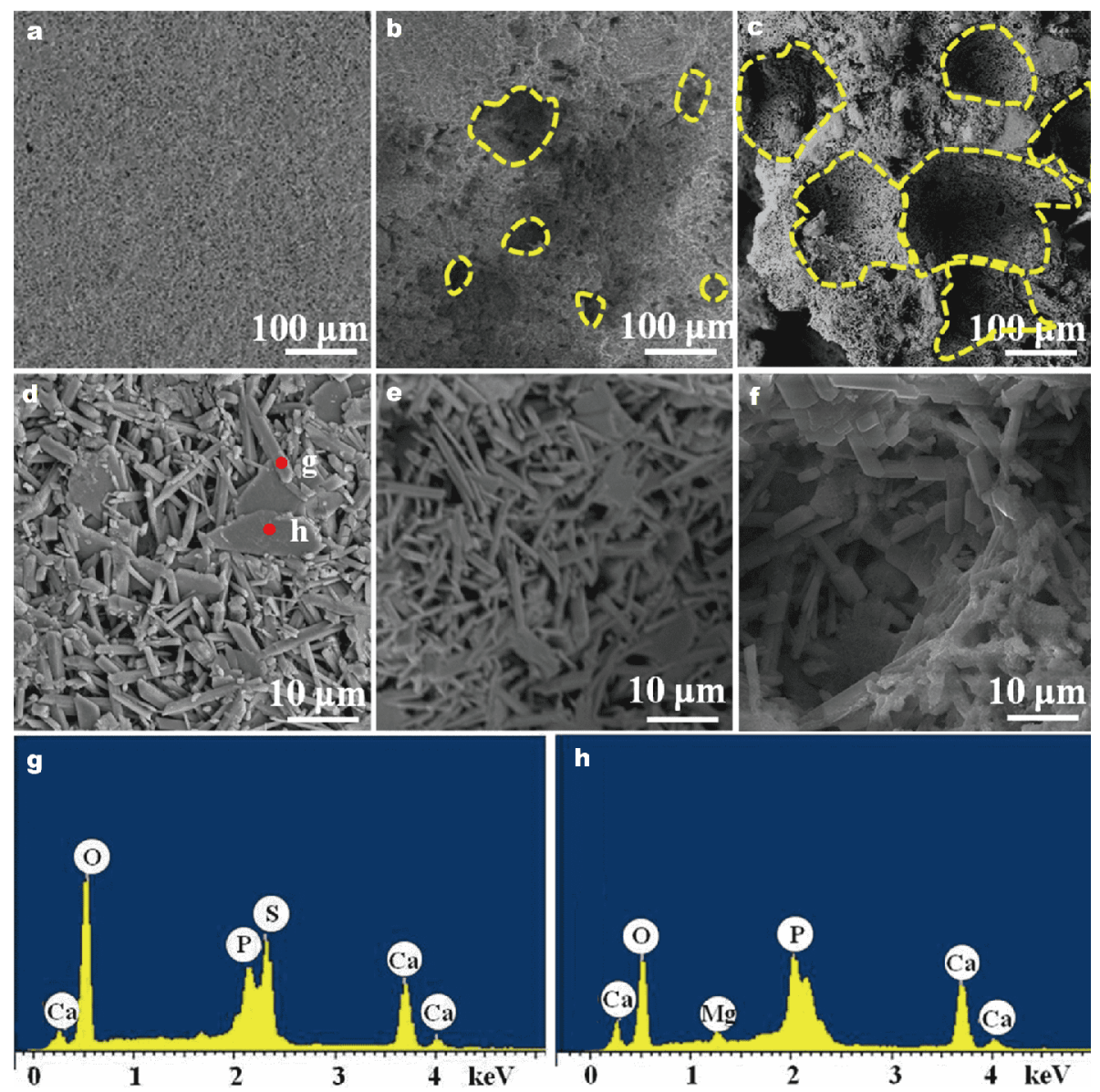

Figure 3 SEM micrographs and EDX results of the injectable composite materials after final setting. SEM micrographs for (a, d) TCMP-CSH, (b, e) TCMP-CSH-1\%Mg, (c, f) TCMP-CSH-5\%Mg; the EDX results of (g) CSD and (h) TCMP particles. Yellow circles in (b, c) represent pores. Red dots in (d) indicate the corresponding EDX positions for $(\mathrm{g}, \mathrm{h})$. 
samples (Fig. S3a, c). A number of macro-pores $(\sim 100-200 \mu \mathrm{m})$ and micro-pores $(<10 \mu \mathrm{m})$ were found in the TCMP-CSH-5\%Mg sample (Fig. S3b, d). The porosity and pore size increased with the increase of $\mathrm{Mg}$ content in the sample. Therefore, $\mathrm{Mg}$ powder can be an effective porogen to modulate the pore size in the injectable composite materials.

EDX mapping for $\mathrm{Ca}, \mathrm{S}, \mathrm{P}$, and $\mathrm{Mg}$ elements of the TCMP-CSH and TCMP-CSH-5\%Mg samples (Fig. 4) confirmed again that CSD comprised of the majority area of the final product, except the pore areas. TCMP granules were homogeneously distributed in the CSD matrix (Fig. 4a). A small amount of Mg granules were found to disperse in the TCMP-CSH-5\%Mg composite matrix (Fig. 4f), which suggested that only a partial amount of $\mathrm{Mg}$ participated in the pore-forming reaction.

\section{In vitro cytotoxicity study}

Cytotoxicity was evaluated following the CCK-8 assay. A distinct increase of cell numbers was observed for the same group with an increase of culture time. These results indicated that the three groups showed no apparent cytotoxicity to the cells at different time periods (Fig. S4). MC3T3-E1 cells adhered to the culture plate and spread well when cultured with regular culture medium or extract from TCMP-CSH, TCMP-CSH- $1 \% \mathrm{Mg}$, and TCMP$\mathrm{CSH}-5 \% \mathrm{Mg}$ samples for 1 day (Fig. 5). $\mathrm{Ca}^{2+}$ concentrations in the extracts of TCMP-CSH, TCMP-CSH- $1 \% \mathrm{Mg}$, and TCMP-CSH-5\%Mg were close to each other, while $\mathrm{Mg}^{2+}$ concentrations obviously increased in the extracts with the increase of $\mathrm{Mg}$ content in the composite materials (Fig. S5). However, no significant difference in cell numbers was found among the various groups (TCMP$\mathrm{CSH}$, TCMP-CSH-1\%Mg, and TCMP-CSH-5\%Mg) at the same time point, suggesting the small amount of $\mathrm{Ca}^{2+}$ and $\mathrm{Mg}^{2+}$ in the extracts have neglectable effects on cell pro-
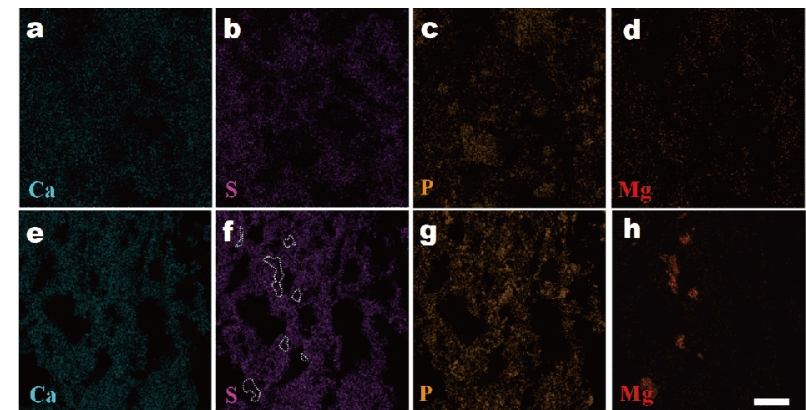

Figure 4 EDX mapping for $\mathrm{Ca}, \mathrm{S}, \mathrm{P}$, and Mg elements in (a-d) TCMP$\mathrm{CSH}$ and (e-h) TCMP-CSH-5\%Mg samples. The white circles show the $\mathrm{Mg}$ granules in the matrix. Scale bar $=200 \mu \mathrm{m}$.
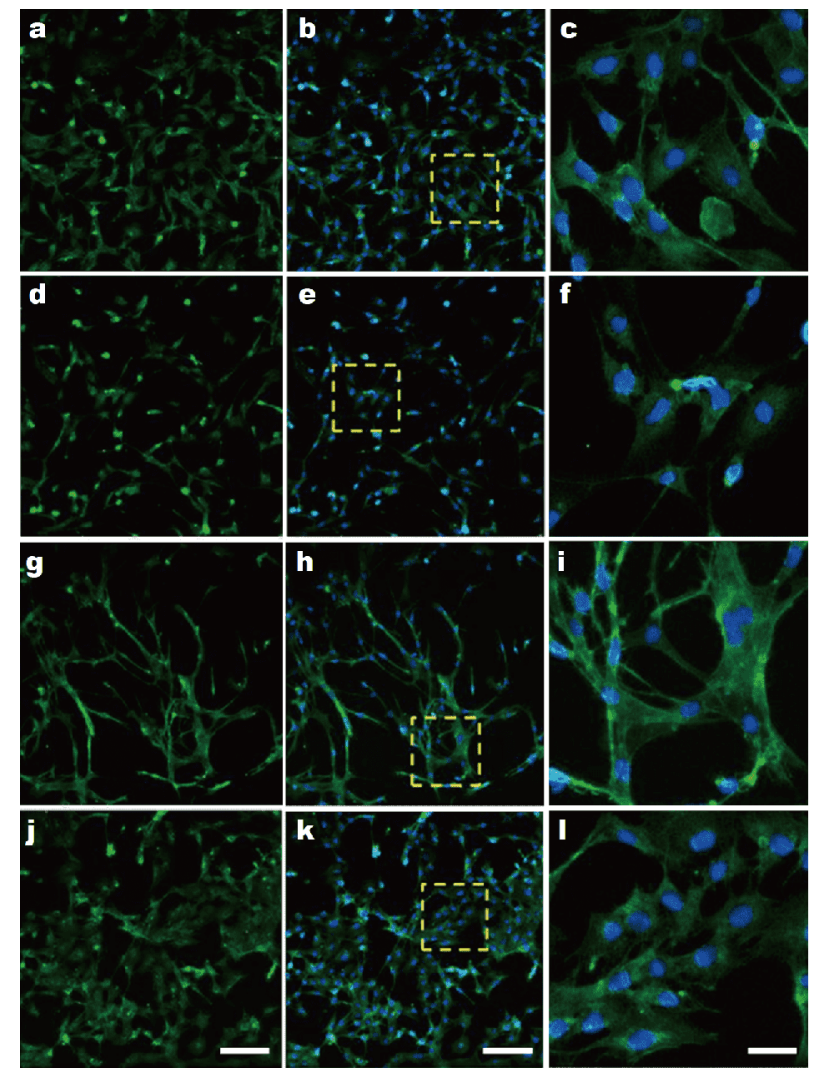

Figure 5 Confocal microscopic images with dual staining of cell nuclei (DAPI, blue) and cytoskeleton F-actin (phalloidin, green) for MC3T3-E1 cells cultured with $(\mathrm{a}-\mathrm{c})$ regular culture medium, and extracts from $(\mathrm{d}-\mathrm{f})$ TCMP-CSH, (g-i) TCMP-CSH- $1 \% \mathrm{Mg}$, and (j-1) TCMP-CSH- $5 \% \mathrm{Mg}$ samples for 1 day. $(c, f, i, l)$ are magnified images for yellow boxes in $(b$, e, h, k), respectively. (a, b, d, e, g, h, j, k) scale bars $=100 \mu \mathrm{m},(\mathrm{c}, \mathrm{f}, \mathrm{i}, \mathrm{l})$ scale bar $=25 \mu \mathrm{m}$.

liferation. Moreover, MC3T3-E1 cells were directly cultured on the TCMP-CSH, TCMP-CSH-1\%Mg and TCMP-CSH-5\%Mg samples to evaluate cellular functions, following the method as previously reported with a minor modification [41]. The cells extended well and exhibited numerous lamellipodia and long filopodia in all experimental groups (Fig. S6). These results suggested that the as-prepared composite materials had no cytotoxicity.

\section{In vivo bone defect repair}

Radiographic X-ray images for the control group, TCMP$\mathrm{CSH}$ and TCMP-CSH-5\%Mg groups post-operation for 10 months are shown in Fig. 6a, e, i. The bone defect areas (Fig. 6e, i) were filled after implantation of TCMP$\mathrm{CSH}$ and TCMP-CSH-5\%Mg samples. The defect in the control group was not fully recovered and remained unfilled, as demonstrated by computed tomography (CT) reconstruction images (Fig. 6b-d). CT reconstruction 


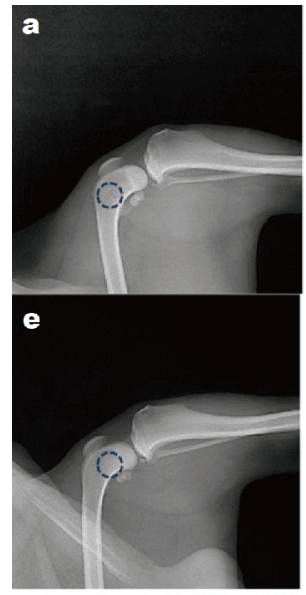

b

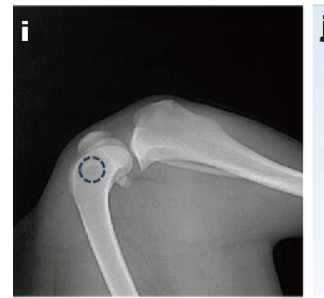

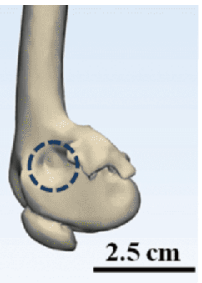
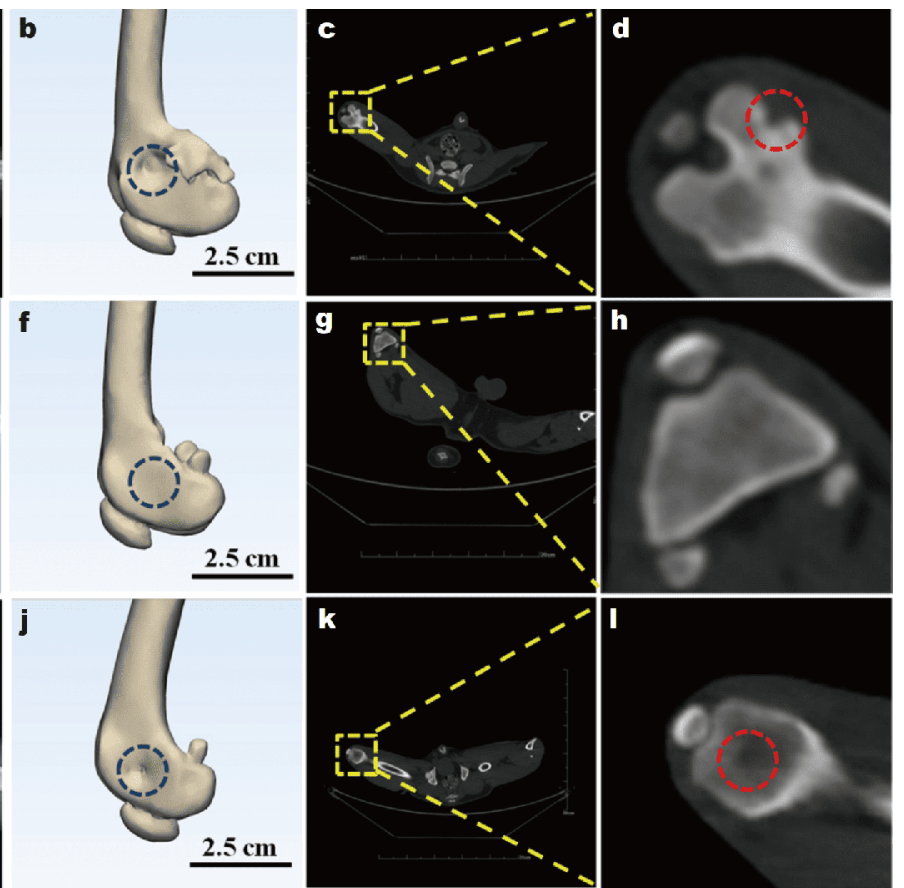

Figure 6 Radiographic X-ray and CT images of the control group (a-d), TCMP-CSH group (e-h), and TCMP-CSH-5\%Mg group (i-l), postoperation for 10 months. Blue circles represent the bone defect areas (a, b, e, f, i, j). (d, h, l) are magnified images for yellow boxes in (c, g, k), respectively. Red circles represent the unrepaired bone defects $(\mathrm{d}, \mathrm{l})$.

images (Fig. $6 f-h, j-1$ ) confirmed that the defects in the TCMP-CSH and TCMP-CSH-5\%Mg groups were nearly completely filled with newly formed bone, although uneven surfaces were observed in the TCMP-CSH-5\% Mg group (Fig. 6j).

Histological H\&E staining for the decalcified sections in the three groups was performed. The defect zone was partially filled by newly formed bone in the control group with discontinuous periosteum (Fig. 7a), and a few osteocytes can be observed in Fig. $7 \mathrm{~b}$ and Fig. S7b. The defect zones were mostly filled with new bone tissue, and thick trabeculae were found in the TCMP-CSH and TCMP-CSH- $\%$ Mg groups. As shown in Fig. $7 \mathrm{c}$ and e, the marrow cavity formed in the TCMP-CSH and TCMP$\mathrm{CSH}-5 \% \mathrm{Mg}$ groups, which can be regarded as a mature bone marker. Numerous osteocytes and erythrocytes were found, especially in the TCMP-CSH and TCMP-CSH$5 \% \mathrm{Mg}$ groups, indicating obvious bone regeneration (Fig. 7d, f and Fig. S7d, f). The histological evaluation also revealed a larger amount of new bone tissue with thicker trabeculae, mature osteocytes, and marrow cavity in the defect areas of TCMP-CSH and TCMP-CSH-5\%Mg groups after 10 months implantation. In contrast, only a few osteocytes and relatively sparse trabeculae could be seen in the control group. New trabeculae were present in all three groups, which were discontinuous in the control group compared with the TCMP-CSH and TCMP-CSH$5 \% \mathrm{Mg}$ groups (Fig. S7a, c, and e). Moreover, the implanted materials in TCMP-CSH and TCMP-CSH-5\%Mg groups could not be found in the histological sections, which were likely thoroughly degraded post-operation for 10 months. In vivo results revealed that both TCMP-CSH and TCMP-CSH-5\%Mg groups can improve osteogenesis and osteointegration compared with the untreated control group.

CPCs and CSCs have been widely used as injectable bone substitutes. However, the former showed relatively slow degradation in vivo, while the latter degraded too fast. For example, CPC blocks $(8 \mathrm{~mm} \times 5 \mathrm{~mm} \times 2 \mathrm{~mm})$ were implanted in beagle alveolar bone defects for 6 months. About $20 \%$ of the CPC samples degraded and the ratio of new bone volume to total volume (BV/TV) was only about $10 \%$, suggesting that slow degradation of CPC samples prevented bone regeneration [42]. On the other hand, CSCs samples showed relatively fast degradation within 4-10 weeks in vivo, and the unfilled defects led to the risk of bone fracture [11,43]. For example, CSCs completely degraded when implanted in the defects ( $8 \mathrm{~mm}$ width) of tibial metaphysis of New Zealand rabbits for one month, and only $34 \%$ of the defect volume was replaced by newly formed bone, leading to further bone fracture [43]. 

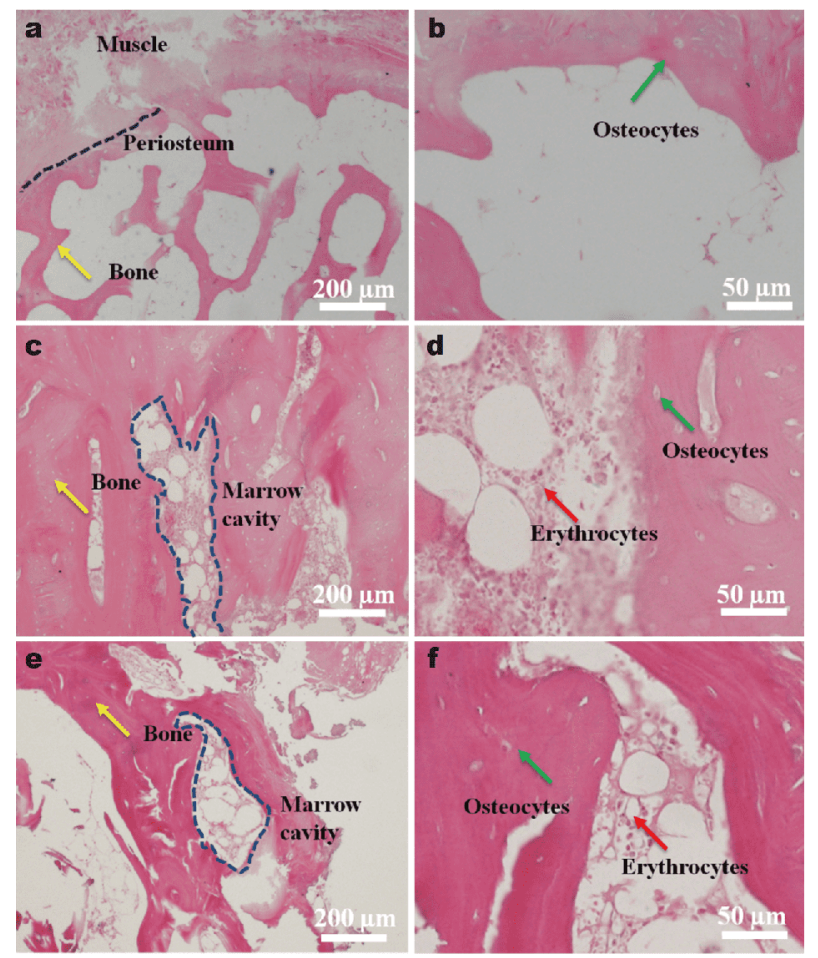

Figure 7 H\&E staining of $(a, b)$ the control group, (c, d) TCMP-CSH group, and (e, f) TCMP-CSH-5\%Mg group after implantation in beagle femoral condyle defects for 10 months. The blue dashed line in (a) shows the periosteum. The yellow arrows in (a, c, e) indicate newlyformed bone. The blue dashed lines in (c, e) show the marrow cavities. The green arrows in $(b, d, f)$ show osteocytes. The red arrows in $(d, f)$ show erythrocytes.

In this study, TCMP and CSH powders were combined together to modulate the degradation rate in vivo. Both TCMP-CSH and TCMP-CSH-5\%Mg samples were completely degraded within 10 months, while the bone defects were nearly fully filled by newly formed bone. No bone fracture was observed for all beagles during the experimental period. Therefore, compared with CPCs and CSCs, the injectable composite materials developed in this study showed suitable degradation rates in vivo, which can facilitate bone regeneration in the case of beagle femoral defect repair.

\section{CONCLUSIONS}

In this study, TCMP nanoparticles with an average particle size of $43.8 \pm 9.0 \mathrm{~nm}$ were synthesized via a wet chemical reaction, which were further mixed with $\mathrm{CSH}$ and PVA to develop the injectable and self-setting composite materials. $\mathrm{Mg}$ granules were utilized as the porogen to produce porous structures with macro-pores (> $100 \mu \mathrm{m})$ during paste preparation. A final setting time
11.7-19.2 min and a compressive strength 2.28 6.33 MPa can be obtained by optimizing the amounts of TCMP, CSH, and Mg in the composite materials. The cytotoxicity assay demonstrated excellent biocompatibility of the composite samples. Moreover, beagle femoral bone defects can be repaired by using the injectable composite materials, which were completely degraded and replaced by newly formed bone after implantation for 10 months. This work reveals that the novel injectable composite materials are promising candidates for bone defect repair in the future.

\section{Received 25 September 2019; accepted 12 December 2019; published online 20 January 2020}

1 Bohner M. Resorbable biomaterials as bone graft substitutes. Mater Today, 2010, 13: 24-30

2 LeGeros RZ. Calcium phosphate-based osteoinductive materials. Chem Rev, 2008, 108: 4742-4753

3 Larsson S, Bauer TW. Use of injectable calcium phosphate cement for fracture fixation: A review. Clin Orthop Relat Res, 2002, 395: 23-32

4 Liu Y, Zheng Y, Hayes B. Degradable, absorbable or resorbablewhat is the best grammatical modifier for an implant that is eventually absorbed by the body? Sci China Mater, 2017, 60: 377391

5 Low KL, Tan SH, Zein SHS, et al. Calcium phosphate-based composites as injectable bone substitute materials: A review. J Biomed Mater Res, 2010, 94: 273

6 Hao F, Qin L, Liu J, et al. Assessment of calcium sulfate hemihydrate-tricalcium silicate composite for bone healing in a rabbit femoral condyle model. Mater Sci Eng-C, 2018, 88: 53-60

7 Moore WR, Graves SE, Bain GI. Synthetic bone graft substitutes. ANZ J Surg, 2001, 71: 354-361

8 Chow LC. Solubility of calcium phosphates. Monogl Oral Sci Basel Karger, 2001, 18: 94-111

9 Goto T, Kojima T, Iijima T, et al. Resorption of synthetic porous hydroxyapatite and replacement by newly formed bone. J Orthop Sci, 2001, 6: 444-447

10 Hibi A, Ishikawa T, Asano M, et al. A study of failed implantation of hydroxyapatite for benign bone tumor. Orthoped Surg, 1994, 45: 1423-1428

11 Bell WH. Resorption characteristics of bone and bone substitutes. Oral Surg Oral Med Oral Pathol, 1964, 17: 650-657

12 Wang ML, Massie J, Perry A, et al. A rat osteoporotic spine model for the evaluation of bioresorbable bone cements. Spine J, 2007, 7: 466-474

13 Masterman D. $K_{\mathrm{sp}}$ determination of calcium sulfate. J Chem Educ, 1987, 64: 408-409

14 Ferguson J, Athanasou N, Diefenbeck M, et al. Radiographic and histological analysis of a synthetic bone graft substitute eluting gentamicin in the treatment of chronic osteomyelitis. J Bone Jt Infect, 2019, 4: 76-84

15 Zhang $\mathrm{T}$, Zhou W, Jia Z, et al. Polydopamine-assisted functionalization of heparin and vancomycin onto microarc-oxidized $3 \mathrm{D}$ printed porous Ti6Al4V for improved hemocompatibility, osteogenic and anti-infection potencies. Sci China Mater, 2018, 61: 579- 
592

$16 \mathrm{Gu} \mathrm{X}$, Wang $\mathrm{F}$, Xie $\mathrm{X}$, et al. In vitro and in vivo studies on asextruded Mg-5.25wt.\%Zn-0.6wt.\%Ca alloy as biodegradable metal. Sci China Mater, 2018, 61: 619-628

17 Park JW, Kim YJ, Jang JH, et al. Osteoblast response to magnesium ion-incorporated nanoporous titanium oxide surfaces. Clin Oral ImPlants Res, 2010, 21: 1278-1287

18 Hung CC, Chaya A, Liu K, et al. The role of magnesium ions in bone regeneration involves the canonical Wnt signaling pathway. Acta Biomater, 2019, 98: 246-255

19 Zhang $\mathrm{X}, \mathrm{Zu} \mathrm{H}$, Zhao $\mathrm{D}$, et al. Ion channel functional protein kinase TRPM7 regulates Mg ions to promote the osteoinduction of human osteoblast via PI3K pathway: In vitro simulation of the bone-repairing effect of Mg-based alloy implant. Acta Biomater, 2017, 63: 369-382

20 Díaz-Tocados JM, Herencia C, Martínez-Moreno JM, et al. Magnesium chloride promotes osteogenesis through Notch signaling activation and expansion of mesenchymal stem cells. Sci Rep, 2017, 7: 7839

21 Tripp RA, Alvarez R, Anderson B, et al. Bioconjugated nanoparticle detection of respiratory syncytial virus infection. Int $\mathrm{J}$ NanoMed, 2007, 2: 117-124

22 Wang $\mathrm{P}$, Zhao L, Liu J, et al. Bone tissue engineering via nanostructured calcium phosphate biomaterials and stem cells. Bone Res, 2014, 2: 14017

23 Zhou C, Xie P, Chen Y, et al. Synthesis, sintering and characterization of porous nano-structured $\mathrm{CaP}$ bioceramics prepared by a two-step sintering method. Ceramics Int, 2015, 41: 4696-4705

24 Fischer J, Prosenc MH, Wolff M, et al. Interference of magnesium corrosion with tetrazolium-based cytotoxicity assays. Acta Biomater, 2010, 6: 1813-1823

25 Matsunaga K, Kubota T, Toyoura K, et al. First-principles calculations of divalent substitution of $\mathrm{Ca}^{2+}$ in tricalcium phosphates. Acta Biomater, 2015, 23: 329-337

26 Polat S, Sayan P. Effects of tricarballylic acid on phase transformation of calcium sulfate hemihydrate to the dihydrate form. Cryst Res Technol, 2017, 52: 1600395

27 Liu X, Wang XM, Chen Z, et al. Injectable bone cement based on mineralized collagen. J Biomed Mater Res, 2010, 94B: 72-79

28 Laurencin CT, Ambrosio AMA, Borden MD, et al. Tissue engineering: orthopedic applications. Annu Rev Biomed Eng, 1999, 1: $19-46$

29 Yu B, Han K, Ma H, et al. Treatment of tibial plateau fractures with high strength injectable calcium sulphate. Int Orthop (SICOT), 2009, 33: 1127-1133

30 Saleh AT, Ling LS, Hussain R. Injectable magnesium-doped brushite cement for controlled drug release application. J Mater Sci, 2016, 51: 7427-7439

31 Lewis G. Injectable bone cements for use in vertebroplasty and kyphoplasty: state-of-the-art review. J Biomed Mater Res, 2006, 76B: 456-468

32 Baino F, Fiume E. Mechanical characterization of $45 \mathrm{~S} 5$ bioactive glass-derived scaffolds. Mater Lett, 2019, 245: 14-17

33 Li X, Niu Y, Guo H, et al. Preparation and osteogenic properties of magnesium calcium phosphate biocement scaffolds for bone regeneration. J Inst, 2013, 8: C07010

34 Zhang J, Liu W, Schnitzler V, et al. Calcium phosphate cements for bone substitution: Chemistry, handling and mechanical properties. Acta Biomater, 2014, 10: 1035-1049

35 Wagoner Johnson AJ, Herschler BA. A review of the mechanical behavior of $\mathrm{CaP}$ and $\mathrm{CaP} /$ polymer composites for applications in bone replacement and repair. Acta Biomater, 2011, 7: 16-30

36 Loh QL, Choong C. Three-dimensional scaffolds for tissue engineering applications: Role of porosity and pore size. Tissue Eng Part B-Rev, 2013, 19: 485-502

37 Chen R, Hunt JA. Biomimetic materials processing for tissueengineering processes. J Mater Chem, 2007, 17: 3974-3979

38 Karageorgiou V, Kaplan D. Porosity of 3D biomaterial scaffolds and osteogenesis. Biomaterials, 2005, 26: 5474-5491

39 Hulbert SF, Young FA, Mathews RS, et al. Potential of ceramic materials as permanently implantable skeletal prostheses. J Biomed Mater Res, 1970, 4: 433-456

40 Babaie E, Lin B, Bhaduri SB. A new method to produce macroporous $\mathrm{Mg}$-phosphate bone growth substitutes. Mater Sci Eng-C, 2017, 75: 602-609

41 Cui W, Song Q, Su H, et al. Synergistic effects of Mg-substitution and particle size of chicken eggshells on hydrothermal synthesis of biphasic calcium phosphate nanocrystals. J Mater Sci Technol, 2020, 36: 27-36

42 Xiao Y, Yin Q, Wang L, et al. Macro-porous calcium phosphate scaffold with collagen and growth factors for periodontal bone regeneration in dogs. Ceramics Int, 2015, 41: 995-1003

43 Orsini G, Ricci J, Scarano A, et al. Bone-defect healing with calcium-sulfate particles and cement: An experimental study in rabbit. J Biomed Mater Res, 2004, 68B: 199-208

Acknowledgements This work was supported by the Key Research Program of Frontier Sciences (QYZDY-SSW-JSC031) from the Chinese Academy of Sciences (CAS), the Key Research and Development Program of Liaoning Province (201703031), Liaoning Science and Technology Project (20170540972) and Shenyang Key R\&D and Technology Transfer Program (Z17-7-023).

Author contributions $\mathrm{Xu} \mathrm{Q}$ and Cao $\mathrm{L}$ prepared the injectable composite materials and conducted the material characterization. Liang $\mathrm{J}$ and Xue $\mathrm{H}$ designed and performed the animal experiments. Liu Y performed HE staining and analyzed the experimental data. Li X, Tian J and Zhang $\mathrm{X}$ conducted the experimental design and result analysis, and revised the manuscript. All authors contributed to the general discussion.

Conflict of interest The authors declare that they have no conflict of interest.

Supplementary information Supplementary data are available online.

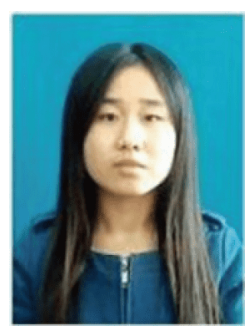

Qian Xu received her master's degree in materials physics and chemistry from the Northeastern University in 2016. She is currently a $\mathrm{PhD}$ student in materials physics and chemistry at the Northeastern University. Her research interest mainly focuses on the development of biomedical materials for orthopaedic applications. 


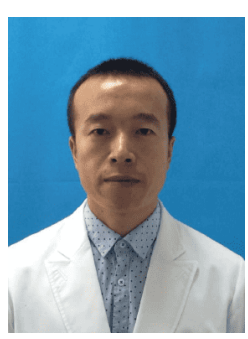

Jiulong Liang received his MD degree in plastic surgery from the Second Military Medical University in 2010. He is currently the director of the Rehabilitation and Reconstruction Center at the General Hospital of the Northern Theater Command. His research interests include microsurgery and auricular reconstruction.

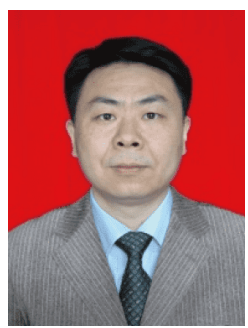

Xiaowu Li received his $\mathrm{PhD}$ degree from the Institute of Metal Research, Chinese Academy of Sciences in 1998. He is currently a professor and head of the Department of Materials Physics and Chemistry, School of Materials Science and Engineering, Northeastern University. His research interest mainly focuses on the mechanical behavior of materials including biomaterials.

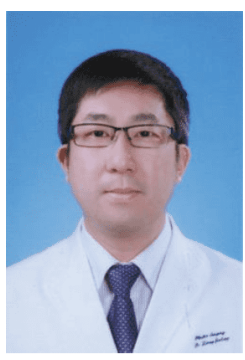

Jing Tian received his MD degree from the Fourth Military Medical University in 2013. He is currently the deputy chief physician and associate professor in the Department of Orthopedics, the General Hospital of the Northern Theater Command. His research interests include traumatic and infectious bone defect repair.

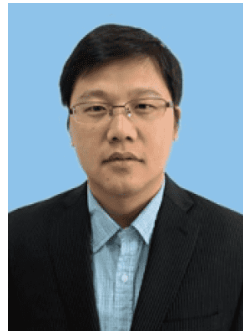

Xing Zhang received his $\mathrm{PhD}$ degree in materials science and engineering from the University of California, San Diego in 2007. He is currently a professor in materials science at the Institute of Metal Research, Chinese Academy of Sciences. His research focuses on biofunctional bone substitutes and polymeric heart valves.

\section{用于骨缺损修复的新型可注射自固化复合材料}

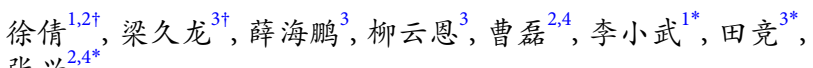
张兴 2 ,

摘要 硫酸钲骨水泥具有良好的骨传导性, 但降解速率快、生物活 性差的缺点限制了其临床应用. 本文将 $\beta$-磷酸三钲纳米颗粒 (粒径 $43.8 \pm 9.0 \mathrm{~nm}$ ) 和半水硫酸钻颗粒 (粒径 $5-21 \mu \mathrm{m}$ ) 混合作为固相, 与 液相聚乙烯醇溶液(5 wt.\%)按优化重量比混匀, 制备了可注射自固 化复合材料. 该材料具有合理的自固化时间(11.7-19.2 min)及适宜 的压缩强度 (2.28-6.33 MPa). 同时, 利用镁颗粒作为成孔剂, 制备出 大孔径(大于 $100 \mu \mathrm{m}$ )的多孔支架. 体外细胞实验显示, MC3T3-E1细 胞伸展良好, 表现出大量的板状伪足和伸展的丝状伪足, 表明该复 合材料无细胞毒性. 将可注射复合材料植入比格犬股骨髁缺损区, 10 个月后骨缺损愈合良好, 表明该材料具有良好的骨缺损修复潜 力. 